\title{
Fetal loss rates and their relation to pregnancy order
}

\author{
EVE ROMAN \\ From the Epidemiological Monitoring Unit, London School of Hygiene and Tropical Medicine, London \\ WC1E $7 H T, U K$
}

SUMMARY Much of the recent controversy surrounding the relation between fetal death and pregnancy order has centred around the appropriateness of different types of analyses. In the present paper the interpretation of various methods are discussed with reference to "real" and "hypothetical" data. The pattern of results obtained when the fetal loss rates of a group of pregnancies are tabulated by pregnancy order was found to depend on the risk and parity distributions of the study population. These two parameters did not, however, appear to affect the within "sibship" or "gravidity" group patterns. These findings support the hypothesis that the frequently observed increase in fetal death rates in pregnancy orders above two could be largely artifactual. It is concluded that in any investigation of reproductive events women, and not their pregnancies, should form the prime unit of analyses.

The nature of the relation between fetal loss and pregnancy order has long been the subject of debate. Much of the controversy has concerned the suitability and interpretation of different methods of study and analyses.

When the fetal loss rates of a group of pregnancies are tabulated by pregnancy order they tend either to increase with increasing pregnancy order ${ }^{1-4}$ or form a $\mathrm{J}$-shaped curve, the lowest rate being found in second or third pregnancies and the rate subsequently increasing with each successive increase in pregnancy order..$^{5-10}$ This pattern has, by tradition, been thought to reflect some biological process - a woman's risk of fetal loss increasing after her second pregnancy with each successive increase in pregnancy order. In this type of "cross sectional" analysis the pregnancy is treated as the prime unit of observation, and it is implicitly assumed that all women experience the same loss rates at the same pregnancy orders. It is, however, generally accepted that some women are at a greater risk of fetal death than others, ${ }^{2411}$ and several authors have pointed out that the fetal loss rates calculated at higher pregnancy orders are likely to be weighted towards high risk women, since women who have fetal losses often compensate for their losses by having more pregnancies than women who have live births. ${ }^{41012-20}$

One method of investigating the association between pregnancy order and fetal loss in the presence of reproductive compensation and risk heterogeneity is to divide the women into groups according to the total number of pregnancies they have had ("gravidity" or "sibship" groups) and tabulate the fetal loss rates of each group by pregnancy order. In this "longitudinal" type analysis the women, not their pregnancies, are the prime units of observation and a reproductive history is required for each woman. Within gravidity or sibship groups the association between fetal loss and pregnancy order is not J-shaped: the fetal loss rates do not increase with each successive pregnancy after the second and fall to their lowest level in the last pregnancy order of the group. ${ }^{10141619}$ This finding has been offered as support for the view that the relation between fetal loss and pregnancy order observed in cross sectional analysis is largely artifactual. ${ }^{101617} 19$ Recently, however, this "method" has been criticised on the grounds that it is not appropriate when birth control is practiced. ${ }^{20-23}$ More specifically, Golding and colleagues used a variety of assumptions to generate a series of hypothetical pregnancy order specific fetal loss rates and concluded that the longitudinal approach to fetal and perinatal risk analysis was grossly misleading. ${ }^{22}{ }^{23}$

In the present paper the problems and biases of various types of analyses are illustrated and discussed with reference to real data and the model of Golding et al. ${ }^{22}$

\section{Data}

The data presented here come from a survey of 
women doctors that has been fully described in the past. $^{91924}$ These data are particularly suitable for studying the relation between fetal loss and pregnancy order. The women were asked to list all their pregnancies and for each pregnancy the outcome and the date of birth or fetal loss was requested. Many of the reproductive histories were complete: the average age of the gravida being 38.4 years. Since the women were not selected because they were pregnant the data do not suffer from the biases associated with obstetric patients. Furthermore, although women doctors are not representative of the general population, they are a socially homogenous group who might be expected to report their reproductive history with some accuracy.

The categories of pregnancy outcome specified in the questionnaire were: live birth, stillbirth, ectopic pregnancy, induced abortion, and spontaneous abortion. Women who reported outcomes other than singleton live birth, stillbirth, or spontaneous abortion and those whose records were in any way incomplete have been excluded from the analyses.

\section{Results}

DA TA

Table 1 shows the numbers of live births, fetal deaths, and fetal death rates (\%) reported by the women doctors. The rates are tabulated by the order of the pregnancy and the gravidity (total pregnancies) of the women. The overall fetal death rates of each pregnancy order and gravidity group are given in the far right hand column and penultimate row respectively. The gravidity distribution of the women is shown in the bottom row.

When all women and pregnancies are combined fetal death rates (far right of table 1) vary with pregnancy order in the classic J-shaped manner: the rates for pregnancy orders one to six being $12.6 \%$, $11 \cdot 5 \%, 13 \cdot 3 \%, 17 \cdot 1 \%, 20 \cdot 9 \%$, and $25 \cdot 3 \%$ respectively. Within gravidity groups (columns), however, the fetal loss rates tend to remain fairly constant until the last pregnancy order of the group when they fall to their lowest point. For example, among the $\mathbf{3 6 9}$ women of gravidity four the fetal loss rates in pregnancy orders one to four were $23.0 \%$, $18 \cdot 4 \%, 16 \cdot 8 \%$, and $9.8 \%$ respectively; and among the 137 wornen of gravidity five the fetal loss rates in pregnancy orders one to five were $27 \cdot 0 \%, 26 \cdot 3 \%$, $29.9 \%, 27.0 \%$, and $13.9 \%$ respectively.

The relation between gravidity and the total fetal loss rate of each gravidity group (penultimate row) is also J-shaped: the rates for gravidities one to seven or more being $11.9 \%, 4.7 \%, 10.6 \% 17.0 \%, 24 \cdot 8 \%$, $29.4 \%$, and $31.6 \%$ respectively. Similarly, in pregnancy order one the fetal death rates vary with gravidity (rows) in a J-shaped fashion: the lowest rate
Table 1 Numbers oflive births $(L B)$, fetal deaths $(F D)$, and fetal death rates (\%)

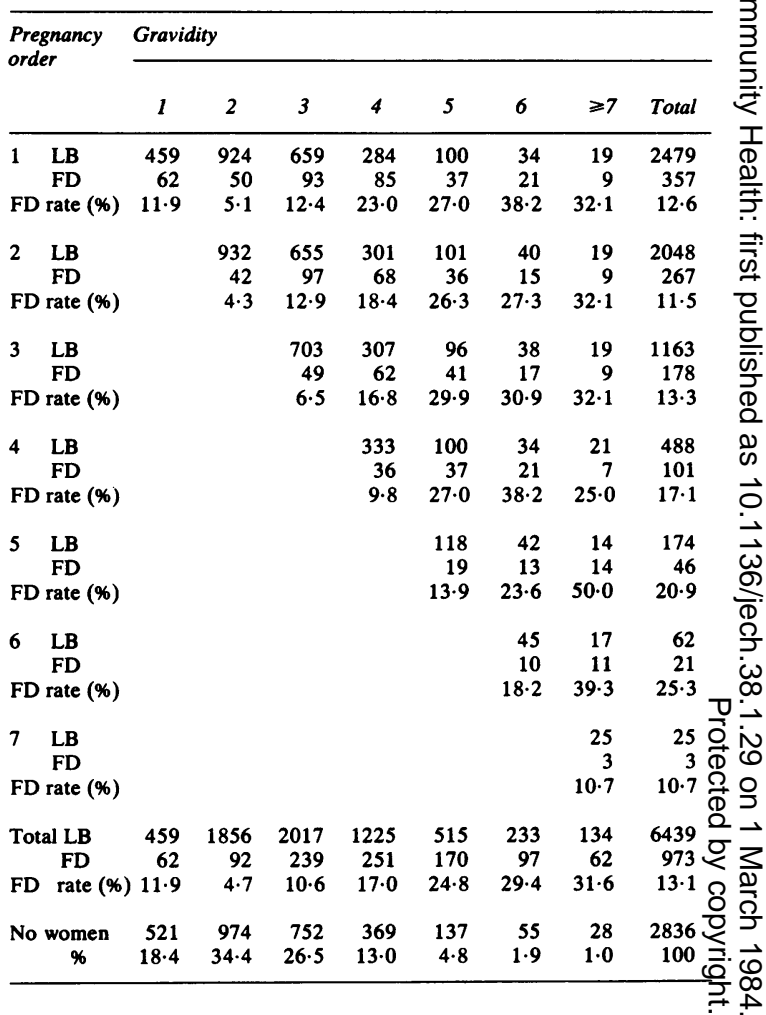

occurring in gravidity two and the rate subsequently increasing with each successive increase in gravidity.

The influences of reproductive compensation and risk heterogeneity on the pattern of rates shown in table 1 are discussed below.

\section{Reproductive compensation}

The low fetal death rate in the last pregnancy order of each gravidity group and at least part of the increase with gravidity in the pregnancy order specific loss rate (table 1) may be explained by reproductive compensation: a woman who has prior fetal loss being more likely to have further pregnancies than a woman who has a prior live birth and thus being more likely to move into a higher gravidity group. That this process was operating within the data presented here may be seen from table 2 , where the continuation rates (\%) following different pregnancy sequences are tabulated by sequence length and the numbers of live births within the sequence: $X$ represents a fetal loss and 0 a live birth. The numbers of women reporting the pregnancy sequences are shown in brackets. The continuation rates following five or more pregnancies are not shown because of small numbers. 
Table 2 Continuation rates (\%) following different pregnancy sequences

\begin{tabular}{|c|c|c|c|c|c|c|}
\hline \multirow{3}{*}{$\begin{array}{l}\text { No of } \\
\text { live births } \\
0\end{array}$} & \multicolumn{5}{|c|}{ Sequence length } & \multirow[b]{2}{*}{ Total } \\
\hline & 1 & 2 & 3 & & 4 & \\
\hline & $X=82.6$ & $X X=81.6$ & $\mathbf{X X X}=73 \cdot 3$ & (15) & $X X X X=25.0$ & $81.6(425)$ \\
\hline 1 & $0=81 \cdot 5(2479)$ & $\begin{array}{l}0 X=84.9 \\
X 0=83.3\end{array}$ & $\begin{array}{l}\mathbf{X X X}=86 \cdot 7 \\
\mathbf{X X X}=85 \cdot 2 \\
\mathbf{X X 0}=\mathbf{7 2 \cdot 0}\end{array}$ & $\begin{array}{l}(30 ; \\
(27) \\
(25)\end{array}$ & $\begin{array}{l}0 X X X=75 \cdot 0 \\
X 0 X X=100 \cdot 0 \\
X X 0 X=66 \cdot 6 \\
X X X 0=71 \cdot 4\end{array}$ & $81 \cdot 8(3041)$ \\
\hline 2 & & $00=50.6(1802)$ & $\begin{array}{l}00 X=65 \cdot 1 \\
0 X 0=47 \cdot 1 \\
X 00=56 \cdot 2\end{array}$ & $\begin{array}{l}(106) \\
(155) \\
(178)\end{array}$ & $\begin{array}{l}00 X X=92.9 \\
0 X 0 X=57.1 \\
0 X X 0=63.6 \\
X 00 X=76.5 \\
\text { X0X0 } 172) \\
X X 00=47.6 \\
\text { (21) } \\
\end{array}$ & $52.0(2351)$ \\
\hline 3 & & & $000=33.4$ & (805) & $\begin{array}{ll}000 X=52.8 & (36) \\
00 X 0=34.5 & (55) \\
0 X 00=30.8 & (52) \\
X 000=32.5 & (83)\end{array}$ & $33.9(1031)$ \\
\hline 4 & & & & & $0000=24 \cdot 5(233)$ & $24.5 \quad(233)$ \\
\hline Total & $81 \cdot 6(2836)$ & $57.9(2315)$ & 43.9( & 1341) & $37.4(589)$ & $63.0(7081)$ \\
\hline
\end{tabular}

$\mathbf{X}=$ Fetal death.

$0=$ Live births.

$(\quad)=$ Number of women

Of the 357 women whose first pregnancy ended in fetal death (X), $82.6 \%$ had a further pregnancy as did $81.5 \%$ of the 2479 women who had a live birth (0). The 521 women who had no more pregnancies are the women of gravidity one (table 1). Thus whether or not the first pregnancy ends in fetal death does not appear to influence the continuation rate: just over $80 \%$ of the 2836 women who had a first pregnancy subsequently had one or more additional pregnancies.

The continuation rates following two, three, or four pregnancies tend to fall as the number of live births increases (columns of table 2). For example, the continuation rates for the pregnancy sequences XX0, X00 and 000 were: $72.0 \%, 56.2 \%$, and $33.4 \%$ respectively. Hence, about two thirds (536) of the 805 women who had three consecutive live births (000) had no further pregnancies. These women contributed to the fetal death rates observed in gravidity three (table 1). About half the women who had one prior loss (X00) and three quarters of those who had two (XX0) moved to a higher gravidity group. Thus the reproductive compensation process causes the pregnancy order specific fetal death rates to increase with increasing gravidity (rows of table 1 ).

The continuation rates of women who have the same numbers of live births are broadly similar (rows of table 2): the rates following zero, one, two, and three live births being about $80 \%, 80 \%, 50 \%$, and $30 \%$, respectively. There, is however, some suggestion that the continuation rates among women who have recurrent losses are low: the rates following one, two, three, and four consecutive fetal deaths being: $82.6 \%, 81 \cdot 6 \%, 73 \cdot 3 \%$, and $25.0 \%$.

The number of women whose pregnancy history ends with a fetal death is comparatively small. For example, the pregnancy sequences $00 \mathrm{X}, 0 \mathrm{X} 0$, and $\mathrm{X} 00$ were reported by 106,155 , and 178 women respectively, and the continuation rates following these sequences were $65 \cdot 1 \%, 47 \cdot 1 \%$, and $56 \cdot 2 \%$ respectively. Hence, only 37 of the 106 women who had a $00 \mathrm{X}$ pregnancy sequence remained in gravidity three as compared with 82 of the 155 women who had a $0 X 0$ sequence and 78 of the 178 women who had an X00 sequence. This trend ensures that within gravidity groups the fetal death rate in the last pregnancy order is low (table 1).

\section{Risk heterogeneity}

That different women suffer different fetal death rates at the same pregnancy orders may be seen from table 3 where the fetal death rates in pregnancy orders one to three are arranged by pregnancy order and previous pregnancy outcome. The combined fetal death rates given on the far right hand side of table 1 are in the bottom row of table 3 .

Of the 2479 women whose first pregnancy ended in a live birth, 1802 had a further live birth in pregnancy order two and 218 a fetal loss: a fetal death rate of $10.8 \%$. Of the 357 women whose first pregnancy ended in fetal death, 246 had a live birth in 
Table 3 Numbers of live births ( $L B)$, fetal deaths (FD), and fetal death rates (\%) arranged by pregnancy order and previous outcome

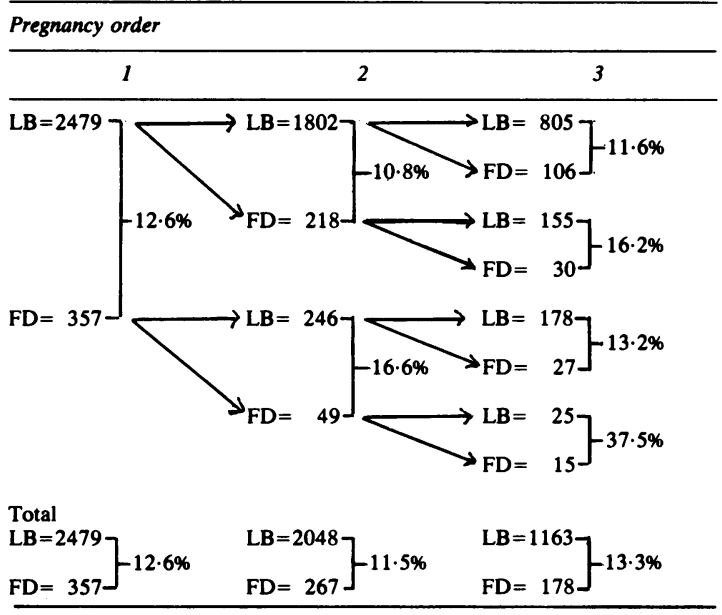

pregnancy order two and 49 a fetal death: a loss rate of $16.6 \%$. These two rates, which combined give an overall fetal death rate of $11.5 \%$, are significantly different $\left(\chi^{2}=8 \cdot 6,1 \mathrm{df}, 0.01<\mathrm{p}<0.05\right)$.

In pregnancy order three the loss rate among the 911 women who had no prior fetal deaths was $11 \cdot 6 \%$. The loss rates in the two groups of women who had one prior fetal death were slightly, although not significantly, greater. The $\mathbf{4 0}$ women whose first and second pregnancies ended in fetal death had a loss rate of $37.5 \%$ in their third pregnancy: this rate is significantly greater than each of the other three rates in pregnancy order three.

In general, within pregnancy orders four and five the results were similar; women with no prior losses had the lowest fetal death rates and women with no prior live births the highest.

These findings imply that within pregnancy orders different women are subject to different risks of fetal death. In the presence of reproductive compensation risk heterogeneity enhances the trend for the pregnancy order specific fetal death rates to increase with increasing gravidity (rows in table 1). Furthermore, variable risk could explain why the combined rate increases with increasing pregnancy order whereas gravidity specific rates do not (columns, table 1): at higher pregnancy orders the combined rate will be weighted towards "high risk" women.

\section{The model}

Recently, the validity of dividing women into gravidity groups has been questioned Golding et al calculated a series of pregnancy order specific fetal death rates using the following assumptions ${ }^{22}$ :

(a) the proportion of pregnancies ending in fetal death was constant at $15 \%$;

(b) the family size preference of the population was $1=15 \%, 2=30 \%, 3=30 \%, 4=15 \%, 5=6 \%$, $6=2 \%, \geqslant 7=2 \%$;

(c) after any one pregnancy, regardless of outcome, $10 \%$ of women became infertile.

Thus $15 \%$ of the population had a fetal death in pregnancy order one and $85 \%$ had a live birth: $19.975 \% \quad(0.85 \times 0.15+(0.85-(0.85 \times 0.15))$ $0 \cdot 1) \times 100$ had one live birth and no further pregnancies - a continuation rate of $76 \%$ : $1.5 \%$ $(0.15 \times 0.1 \times 100)$ had one fetal death and no further pregnancies-a continuation rate of $90 \%$ : and so on.

Models of fetal death can, however, be made to produce a large number of patterns and the conclusions drawn depend largely on how the results are presented. Table 4 gives the hypothetical fetal death rates generated by the above model.

The pattern of rates shown in table 4 is in many ways similar to that presented in table 1: at any given pregnancy order the rate rises with increasing gravidity and within gravidity groups it remains constant before falling to its lowest level in the last pregnancy order. In the "real" data (table 1) these trends were interpreted here and elsewhere ${ }^{1619}$ in terms of reproductive compensation; and in the model (table 4) they are directly caused by the reproductive compensation assumption $(b)$. The assumption ( $a$ ) of a constant fetal death rate at each pregnancy order is thus reflected within gravidity groups. If the data shown in table 4 were real one could correctly conclude that the fetal loss rates did not alter, on average, with pregnancy order and that

Table 4 Fetal loss rates (\%) generated using the (1982) assumptions of Golding et al ${ }^{22}$

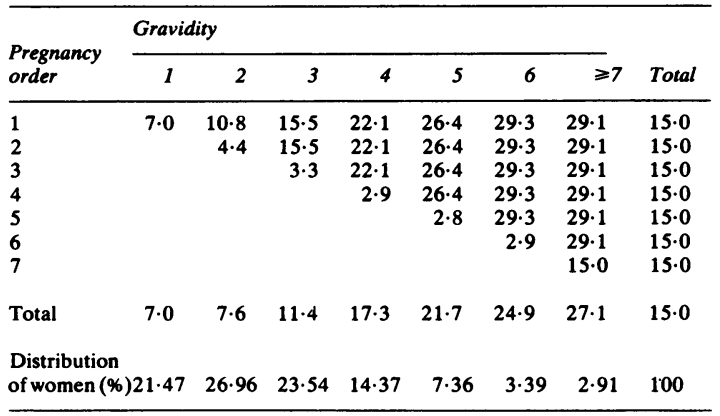


reproductive compensation was probably operating within the population being studied. If, on the other hand, the risk of fetal death varied with pregnancy order in a systematic fashion (either increasing or decreasing) the within gravidity group rates would consistently show this pattern.

Golding et al noted that when women with seven or more pregnancies were excluded from the analysis shown in table 4 the overall pregnancy order specific rates fell as pregnancy order increased (table 5) ${ }^{22}$; and on the basis of this result they concluded that within gravidity group analyses were misleading. Their results, however, simply reflect the fact that a reproductive compensation assumption $(b)$ was built into their model. The exclusion of high gravidity women only affects the combined "cross sectional" rate (table 5), it does not influence the within gravidity group rates (table 4). Hence, an examination of these rates would not, as the authors claimed, have led to the erroneous conclusion that fetal death risk fell as pregnancy order increased.

Table 5 Pregnancy order specific fetal loss rates: (a) all and (b) women with fewer than seven pregnancies from Golding et al a2 $^{2}$

\begin{tabular}{lllll}
\hline & \multicolumn{4}{l}{ Pregnancy order } \\
\cline { 2 - 5 } & 1 & 2 & 3 & 4 \\
\hline (a) & 15.0 & 15.0 & 15.0 & 15.0 \\
(b) & 14.6 & 14.5 & 14.1 & 13.4 \\
\hline
\end{tabular}

One difference between the hypothetical rates (table 4) and the real rates (table 1) could stem from the model's assumption of equal risk. The addition of a variable risk assumption to the model of Golding $e t$ $a l^{22}$ causes the combined rate to rise in the absence of any within gravidity group change. As an example, table 6 shows the rates generated by assuming that $90 \%$ of women experience a loss rate of $15 \%$ and $10 \%$ a loss rate of $50 \%$. The $50 \%$ loss rates are shown in table 7. If the hypothetical rates given in table 6 come from a real population the inconsistency between the combined and within gravidity group pattern could lead to a heterogeneity of risk factor hypothesis. If however, the combined rates were presented on their own one could mistakenly postulate that fetal death risk increased with increasing pregnancy order.

In table 1 both the pregnancy order specific combined fetal death rates (far right hand column) and the gravidity specific fetal death rates (rows) exhibit a J-shaped pattern. These trends depend on the family size assumption $(b)$ and can be reproduced without assuming that fetal death rates vary with
Table 6 Fetal loss rates (\%) generated by adding risk heterogeneity to the assumptions of Golding et al $22.15 \%$ loss risk for $90 \%$ of the population and a 50\% loss risk for $10 \%$

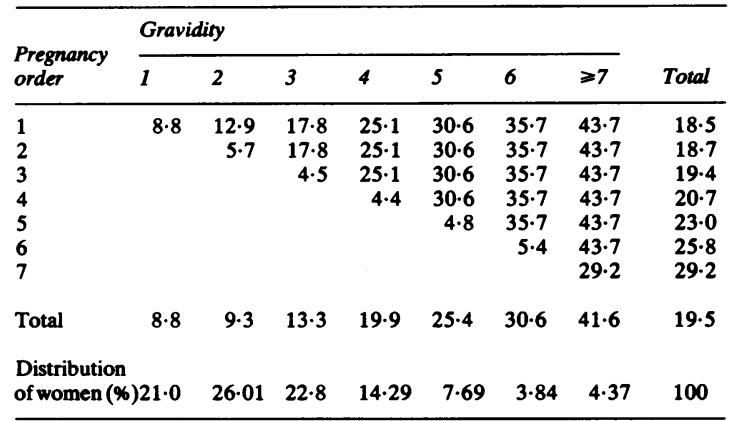

Table 7 Fetal loss rates (\%) generated using the assumptions of Golding et al ${ }^{22}$ and fetal loss risk of $50 \%$

\begin{tabular}{|c|c|c|c|c|c|c|c|c|}
\hline \multirow{2}{*}{$\begin{array}{l}\text { Pregnancy } \\
\text { order }\end{array}$} & \multicolumn{7}{|c|}{ Gravidity } & \multirow[b]{2}{*}{ Total } \\
\hline & 1 & 2 & 3 & 4 & 5 & 6 & $\geqslant 7$ & \\
\hline $\begin{array}{l}1 \\
2 \\
3 \\
4 \\
5 \\
6 \\
7\end{array}$ & 29.9 & $\begin{array}{l}43 \cdot 2 \\
23 \cdot 9\end{array}$ & $\begin{array}{l}48.6 \\
48.6 \\
20.6\end{array}$ & $\begin{array}{l}53 \cdot 5 \\
53 \cdot 5 \\
53 \cdot 5 \\
18 \cdot 3\end{array}$ & $\begin{array}{l}57.4 \\
57.4 \\
57.4 \\
57.4 \\
17.0\end{array}$ & $\begin{array}{l}60.5 \\
60.5 \\
60 \cdot 5 \\
60 \cdot 5 \\
60.5 \\
16 \cdot 0\end{array}$ & $\begin{array}{l}65 \cdot 3 \\
65 \cdot 3 \\
65 \cdot 3 \\
65 \cdot 3 \\
65 \cdot 3 \\
65 \cdot 3 \\
50 \cdot 0\end{array}$ & $\begin{array}{l}\mathbf{5 0} \\
\mathbf{5 0} \\
\mathbf{5 0} \\
\mathbf{5 0} \\
\mathbf{5 0} \\
\mathbf{5 0} \\
\mathbf{5 0}\end{array}$ \\
\hline Total & 29.9 & 33.5 & $39 \cdot 3$ & $44 \cdot 7$ & 49.3 & 53.1 & 63.1 & 50 \\
\hline $\begin{array}{l}\text { Distribution } \\
\text { of women ( } \%\end{array}$ & $16 \cdot 75$ & $17 \cdot 43$ & $16 \cdot 15$ & 13.58 & $10 \cdot 64$ & $7 \cdot 92$ & $17 \cdot 55$ & 100 \\
\hline
\end{tabular}

pregnancy order. As an example, table 8 shows the fetal death rates generated by altering the family size preference of the hypothetical population as follows: $1=5 \%, 2=30 \%, 3=40 \%$, and $\geqslant 4=25 \%$. In this model only a few women stop after having had one child and comparatively more desire three. This modification causes the gravidity specific fetal death rates (rows) to vary with gravidity in a J-shaped manner, the lowest rate occurring in gravidity two (table 8). The fetal death rates generated by assuming that $90 \%$ of the population conforms to this pattern and $10 \%$ to that shown in table 7 are given in table 9. This model combines risk heterogeneity with variable reproductive compensation: it being assumed that "high risk" women, who are more likely to experience recurrent losses, may have to settle for smaller families than "low risk" women. This causes the combined cross sectional fetal death rates to vary with pregnancy in a J-shaped fashion; the rates for pregnancy orders one to four being $18 \cdot 5$, $18 \cdot 2,18 \cdot 8$, and $20 \cdot 3$ respectively. 
Table 8 Fetal loss rates generated using the assumptions of Golding et al ${ }^{22}$ but a family size preference of : $1=5 \%$, $2=30 \%, 3=40 \%, \geqslant 4=25 \%$

\begin{tabular}{llllll}
\hline $\begin{array}{l}\text { Pregnancy } \\
\text { order }\end{array}$ & \multicolumn{2}{l}{ Gravidity } & & & \\
\cline { 2 - 5 } & 1 & 2 & 3 & $\geqslant 4$ & Total \\
\hline 1 & 10.8 & 7.0 & 12.6 & 26.1 & 15.0 \\
2 & & 4.8 & 12.6 & 26.1 & 15.0 \\
3 & & & 3.1 & 26.1 & 15.0 \\
4 & 10.8 & 5.9 & 9.4 & 23.3 & 15.0 \\
Total & & & & & 15.0 \\
$\begin{array}{l}\text { Distribution } \\
\text { of women (\%) }\end{array}$ & 13.82 & 26.68 & 28.66 & 30.84 & 100 \\
\hline
\end{tabular}

Table 9 Fetal loss rates (\%) generated by combining two hypothetical populations (a) and (b) in the proportions 9:1. Population (a) having a 15\% fetal death rate and a family size preference of :1 $1=5 \%, 2=30 \%, 3=40 \%, \geqslant 4=25 \%$ and (b) having a 50\% fetal death rates and a family size preference of: $1=15 \%, 2=30 \%, 3=30 \%$ and $\geqslant 4=25 \%$

\begin{tabular}{llrrll}
\hline \multirow{2}{*}{$\begin{array}{l}\text { Pregnancy } \\
\text { order }\end{array}$} & \multicolumn{2}{l}{ Gravidity } & & & \\
\cline { 2 - 5 } & 1 & 2 & 3 & $\geqslant 4$ & Total \\
\hline 1 & 13.1 & 9.1 & 14.7 & 31.2 & 18.5 \\
2 & & 6.1 & 14.7 & 31.2 & $\begin{array}{l}18.2 \\
3\end{array}$ \\
4 & & & 4.1 & 31.2 & 18.8 \\
Total & 13.1 & 7.6 & 11.2 & 28.5 & 18.7 \\
$\begin{array}{lllll}\text { Distribution } \\
\text { of women (\%) }\end{array}$ & 14.11 & 25.76 & 27.41 & 32.72 & 100 \\
\hline
\end{tabular}

\section{Discussion}

The findings presented here support the hypothesis that the frequently observed increase in fetal death rates in pregnancy orders above two could be largely due to the interaction of two variables-reproductive compensation and risk heterogeneity. The observed relation between fetal death and pregnancy order in a cross section of pregnancies will thus depend on the distribution of these two factors.

That different women are probably subject to different, but in many cases relatively constant, risks of fetal death does not, however, mean that an individual woman's risk cannot change. Although the magnitude and manner of the increase may be disputed, most workers agree that the risk of fetal death among women who became pregnant towards the end of their reproductive lives is comparatively high. ${ }^{122528}$ Moreover, an individual's risk of fetal death can obviously be altered by a past exposure or event, one such event being a prior pregnancy. This pregnancy could in some cases lead to a woman being more likely to have a miscarriage or a stillbirth in a subsequent pregnancy. On the other hand, the ability of some women to deliver a healthy term baby may be improved by the experience of a previous pregnancy. It is, for example, widely believed that first pregnancies are, if anything, at a higher risk than second. Moreover, the fetal death rates of women who have no prior fetal deaths are usually found to be comparatively low, Indeed, the current climate of opinion seems to be moving towards the view that in most populations increasing pregnancy order, per se, does not, on average, cause an increase in fetal death risk. Hence, much of the controversy concerning the interpretation of fetal death rates and their relation to pregnancy order is largely academic. It is, however, clear from many studies that variation between women should be taken fully into account when investigating reproductive events or variables, such as gestational age, birth weight, or pregnancy interval. Dividing women into gravidity or sibship groups is only one way of analysing reproductive history data. The suitability and interpretation of this, or alternative, methods depends largely on the nature of the investigation and the data that are available.

Previous work by Valerie Beral and Eva Alberman has contributed to this paper. I am grateful to them for their continued interest and support.

\section{References}

${ }^{1}$ Stevenson A, Dudgeon M, McClure H. Observations on the results of pregnancies in women resident in Belfast. Ann Hum Genet 1959; 23: 395-411.

${ }^{2}$ Warburton D, Fraser FC. Spontaneous abortion risks in man: data from reproductive histories collected in medical genetics unit. Hum Genet 1964; 16: 1-25.

${ }^{3}$ Shapiro S, Jones EW, Densen PM. A life table of pregnancy terminations and correlates of fetal loss. Milbank Mem Fund 1962; 40: 7-29.

${ }^{4}$ Léridon H. Facts and antifacts in the study of intra-uterine mortality: a reconsideration from pregnancy histories. Population Studies 1976; 30: 319-35.

${ }^{5}$ Yerulshamy J, Bierman JM, Kemp DH, Connor A, French FE. Longitudinal studies of pregnancy on the island of $\mathrm{Kwai}$, territory of Hawaii. I. Analysis of previous reproductive history. Am J Obstet Gynecol 1956; 71: 8-86.

${ }^{6}$ Baird D, Thompson A. General factors underlying perinatal mortality. In: Butler $\mathbf{N}$, Alberman $\mathrm{E}$, eds. Perinatal problems: the second report of the British perinatal mortality survey. London: Livingstone, 1969.

${ }^{7} \mathrm{Jain}$ AK. Fetal wastage in a sample of Taiwanese women. Milbank Mem Fund $Q$ 1969; 47: 297-306.

8 James WH, Stillbirth and birthorder. Ann Hum Genet 1968; 32: 151-72.

${ }^{\circ}$ Roman E, Doyle P, Beral V, Alberman E, Pharoah P. Fetal loss, gravidity and pregnancy order. Early Hum Dev 1978; 2: 131-38.

${ }^{10}$ Bakketeig LS, Hoffman HJ. Perinatal mortality by birth order within cohorts based on sibship size. $\mathrm{Br}$ Med J 1979; ii: 693-6. 
${ }^{11}$ Strobino BR, Kline J, Shrout P, Stein Z, Susser M, Warburton D. Recurrent spontaneous abortion: definition of a syndrome. In: Hook EB, Parker IH, eds. Human embryonic and fetal death. New York: Academic Press, 1980: 315-29.

12 James WH. On the possibility of segregation in the propensity to spontaneous abortion in the human female. Ann Hum Genet 1961; 25: 207-13.

${ }^{13}$ Resseguie LJ. Influence of age, birth order and reproductive compensation on stillbirth ratios. $J$ Biosoc Sci 1973; 5: 443-52.

${ }^{14}$ Billewicz WZ. Some implications of self-selection for pregnancy. Br J Prev Soc Med 1973; 27: 49-52.

${ }^{15}$ James WH. Spontaneous abortion and birth order. $J$ Biosoc Sci 1974; 6: 23-41.

${ }^{16}$ Roman E, Alberman E, Beral V. Pregnancy order and reproductive loss [Letter]. Br Med J 1980; 280: 715.

${ }^{17}$ Bakketeig LS, Hoffman HJ. Pregnancy order and reproductive loss [Letter). Br Med J 1980; 280: 716.

${ }^{18}$ Balleweg JA. Death loss, fetal wastage and completed family size. J Biosoc Sci 1982; 14: 309-18.

${ }^{19}$ Roman E. Spontaneous abortion and its relationship to various maternal and obstetric factors. London: London University, 1982. (PhD thesis.)

${ }^{20}$ Wilcox AJ, Gladen BC. Spontaneous abortion: the role of heterogeneous risk and selective fertility. Early Hum Dev 1982; 7: 165-78.

${ }^{21}$ Mantel N. Perinatal mortality by birth order [Letter]. $\mathrm{Br}$ Med J 1979; ii: 1147.

${ }^{22}$ Golding J, Vivian S, Newcombe R. Fetal loss, gravidity and pregnancy order: is the truncated cascade analysis valid? Early Hum Dev 1982; 6: 71-6.

${ }^{23}$ Golding J; Butler N, Newcombe R. Analysis of completed reproductive histories: a cautionary tale. $J$ Epidem Community Health 1983; 37: 78-81.

${ }^{24}$ Pharaoh POD, Alberman E, Doyle P. Outcome of pregnancy among women in anaesthetic practice. Lancet 1977; i: 34-6.

${ }^{25}$ Kline J, Shrout PE, Stein ZA, Susser M, Weiss M. An epidemiological study of the role of gravidity in spontaneous abortion. Early Hum Dev 1979; 1: 345-56.

${ }^{28}$ Resseguie LJ. Comparison of longitudinal and crosssectional analyses: maternal age and stillbirth ratio. Am J Epidemiol 1976; 103: 551-9. 